\title{
A Proposed High-Gain Observer for a Class of Nonlinear Fractional-Order Systems
}

\author{
Dorsaf Etlili (iD, Atef Khedher (D), and Ayachi Errachdi (i) \\ Tunis El Manar University, National Engineering School of Tunis, Department of Electrical Engineering, Le Belvédère B.P. 37, \\ Tunis 1002, Tunisia \\ Correspondence should be addressed to Dorsaf Etlili; dorsaf.etlili@enit.utm.tn
}

Received 26 April 2021; Revised 8 July 2021; Accepted 14 July 2021; Published 26 July 2021

Academic Editor: Abdesselem Boulkroune

Copyright () 2021 Dorsaf Etlili et al. This is an open access article distributed under the Creative Commons Attribution License, which permits unrestricted use, distribution, and reproduction in any medium, provided the original work is properly cited.

This paper proposes a high-gain observer for a class of nonlinear fractional-order systems. Indeed, this approach is based on Caputo derivative to solve the estimation problem for nonlinear systems. The proposed high-gain observer is used to estimate the unknown states of a nonlinear fractional system. The use of Lyapunov convergence functions to establish stability of system is detailed. The influence of different fractional orders on the estimation is presented. Ultimately, numerical simulation examples demonstrate the efficiency of the proposed approach

\section{Introduction}

In recent years, fractional calculus deals with derivatives and integrations of arbitrary order $[1,2]$ and has found many applications in different areas of physics, applied mathematics, and technology. Due to their characterization by fractional-order equations, several studies show that certain dynamics systems are governed by differential equations with noninteger derivatives. The use of classical models based on an integer derivation is therefore not appropriate. Therefore, models based on noninteger differential equations have been developed.

For these reasons, there are several analyses for fractional derivatives, such as the definition of Caputo [3], Riemann-Liouville, and Grünwald-Letnikov [4].

It can be seen that the description of some systems is more accurate when the fractional derivative is used. For example, the heat transfer process modelling for photovoltaic/thermal hybrid system [5], which is defined by heterogeneous media due to the multilayers that make up the system, can be characterized by a fractional-order partial differential equation. The applications are numerous, whether in electricity, chemistry, or signal processing [6-9].

The main problem for the automation researcher is to represent these physical processes with sufficient estimation and a simple structural model. The difficulty lies then between the fidelity of the model with respect to the real process and the transformation of this model to a mathematically exploitable form.

To overcome these difficulties, different designs of observers are developed. An observer that can be described as the association of a physical sensor with an algorithm that provides, from the measurements provided by the physical sensor and the inputs applied to the system, online estimates of the different state variables.

The synthesis of observer for linear systems is completely characterized by well-established necessary and sufficient conditions. Indeed, the first works on observer, published around the sixties by Kalman [10] and Luenberger [11], are interested in time-invariant linear systems.

However, most of the industrial processes have nonlinear behaviors which encouraged researchers to develop nonlinear observers with applications such as extended Kalman filter [12], high-gain observers [13], or sliding mode estimators $[14,15]$.

More recently, several studies have tried to solve the observer design problem for noninteger-order nonlinear systems. For instance, in [16-18], researchers paid attention to nonlinear observer designs for fractional chaotic systems.

In [19], Boroujeni and Momeni focused on a nonfragile nonlinear fractional-order observer design, and they 
proposed the indirect application of Lyapunov to find LMIbased nonfragile fractional-order observer gain for a class of Lipschitz nonlinear systems using continuous frequency distribution.

Fractional high-gain observer is an algorithm processed by several authors to solve the problem of estimation $[20,21]$. It was shown that when using high-gain observers, the state estimate converges to the actual state rapidly. It was exhibited that when high-gain observers are used in output feedback control, they can regain the performance of the state feedback controller.

In [22], a novel high-gain observer with Mittag-Leffler function was proposed.

In [23, 24], a sliding mode fractional observer for nonlinear systems was developed. In consideration of its performance and its robustness to uncertainty and perturbations, the sliding mode observer is attracting the interest of researchers in fractional-order systems.

Most of these approaches of fractional-order nonlinear systems are based on the Lyapunov stability [25-27].

In this paper, a high-gain observer is proposed for fractional-order nonlinear systems.

This paper is organized as follows. After presentation of an introduction in Section 1, some preliminaries about the fractional-order nonlinear systems are presented in Section 2. The fractional-order high-gain observer technique is detailed in Section 3. Section 4 presents a stability study of the proposed high-gain observer. In Section 5, simulation results and remarks are provided. Section 6 gives some conclusions.

\section{Preliminaries}

In this section, some definitions and lemmas associated with fractional calculus are presented. Indeed, we focus on the definition of Caputo fractional derivative. Fractional calculus is a generalization of integration and differentiation to the fundamental noninteger-order operator.

Definition 1. The gamma function is defined as

$$
\Gamma(\alpha)=\int_{0}^{+\infty} e^{-t} u^{\alpha-1} \mathrm{~d} u .
$$

Definition 2. The Caputo fractional derivative is defined as

$$
{ }_{\mathbf{a}}^{\mathbf{C}} \mathbf{D}_{\mathbf{t}}^{\alpha} f(t)=\frac{1}{\Gamma(r-\alpha)} \int_{a}^{t}(t-\tau)^{r-\alpha-1} f^{(r)}(\tau) \mathrm{d} \tau
$$

with $r$ is a positive integer verifying the inequality $(r-1)<\alpha<r$. According to the Caputo definition, $\mathbf{D}_{t}^{\alpha} f(t)$, denotes the fractional-order derivative $\alpha$ of the function $f(t)$ between $a$ and $t$.

However, when using the Caputo derivative, the initial conditions take the same form as that integer-order differential equations.
Furthermore, the Mittag-Leffler function, which has achieved importance in the solution of fractional-order systems, can be expressed as follows:

$$
E_{\alpha}(z)=\sum_{k=0}^{+\infty} \frac{z^{k}}{\Gamma(\alpha k+1)},
$$

where $0<\alpha<1$.

The Mittag-Leffler function plays a role similar to the exponential function; it develops naturally when solving fractional differential equations.

In this paper, we focus on the fractional-order nonlinear systems described by the following equation:

$$
\left\{{ }^{c} \mathbf{D}_{\mathbf{t}}^{\alpha} x(t)=A x+B u+f(x, u), y(t)=C x(t),\right.
$$

where $x \in \mathbb{R}^{n}$ is the state, $u \in \mathbb{R}^{m}$ is the input, $f \in C\left(\mathbb{R}^{n} \times \mathbb{R}^{m}, \mathbb{R}^{n}\right)$, and $A \in \mathbb{R}^{n \times n}, \quad B \in \mathbb{R}^{n \times m}$, and $C \in \mathbb{R} q \times n$ are system matrices.

Definition 3. Moreover, $f: \mathbb{R}^{n} \longrightarrow \mathbb{R}^{m}$ is a nonlinear function that is Lipschitz with a Lipschitz constant $d$. This is a necessary and sufficient condition for the stability of a nonlinear system, defined by

$$
\left\|f\left(x_{1}\right)-f\left(x_{2}\right)\right\| \leq d\left\|x_{1}-x_{2}\right\| .
$$

Definition 4. The fractional system (4) is said to be MittagLeffler stable, if for any initial condition $x_{0}$, at any initial time $t_{0}$, its solution satisfies

$$
\left\|x\left(t, x_{0}\right)\right\| \leq\left[d\left(\left\|x_{0}\right\|\right) E_{\alpha}\left(\lambda\left(t-t_{0}\right)^{\alpha}\right)\right]^{(1 / b)},
$$

where $b>0$, and satisfies $d(0)=0$.

Then, from the definition of caputo fractional derivative, we can obtain the following lemmas.

Theorem 1 (see [28]). Let $\boldsymbol{x}=0$ be an equilibrium point of system (4), and $x_{0}=x\left(t_{0}\right)$, where $\alpha \in(0,1)$.

Let $V(t, x(t)):[0, \infty) \times \mathbb{R}^{n} \longrightarrow \mathbb{R}$ be a continuously differentiable function and locally Lipschitz with respect to $x$, such that

$$
\begin{aligned}
m_{1}\|x\|^{a} \leq V(t, x(t)) & \leq m_{2}\|x\|^{a b}, \\
{ }^{c} D_{t}^{\alpha} V(t, x(t)) & \leq-m_{3}\|x\|^{a b},
\end{aligned}
$$

where $t \geq 0, \alpha \in(0,1)$, and $m_{1}, m_{2}, m_{3}, a, b$ are arbitrary positive constants. Then, $x=0$ is Mittag-Leffler stable.

Lemma 1 (see [29]). Let $x \in \mathbb{R}^{n}$ be a vector of differential functions. Then, for any time instant $t \geq 0$, the following relationship applies:

$$
{ }^{c} D_{t}^{\alpha}\left(x(t)^{T} P x(t)\right) \leq 2 x(t)^{T} P^{c} D_{t}^{\alpha} x(t)
$$

$\forall \alpha \in(0,1), \forall t \geq t_{0}$, where $P \in \mathbb{R}^{n \times n}$ is a constant, square, symmetric, and positive definite matrix. 


\section{Fractional High-Gain Observer}

This paper considers the problem of designing a continuoustime observer for fractional systems.

A fractional high-gain observer (FHGO) is proposed to estimate the fractional-order system (4) described by the following equation:

$$
\begin{array}{r}
{ }^{c} \mathbf{D}_{\mathrm{t}}^{\alpha} \widehat{x}(t)=A \widehat{x}+B u+f(\widehat{x}, u) \\
-\theta \Delta_{\theta}^{-1} S^{-1} C^{T}(C \widehat{x}-y),
\end{array}
$$

where $\hat{x} \in \mathbb{R} n$ is the state estimation and $\Delta_{\theta}$ is a diagonal matrix given as follows:

$$
\Delta_{\theta}=\operatorname{diag}\left[I_{p} \frac{1}{\theta} I_{p} \cdots \frac{1}{\theta^{q-1}} I_{p}\right] .
$$

$S$ is a positive definite matrix solution of Lyapunov equation (11):

$$
\theta S+A^{T} S+S A-C^{T} C=0
$$

and the vector $S^{-1} C^{T}$ can be expressed as follows:

$$
S^{-1} C^{T}=\left(\begin{array}{c}
C_{q}^{1} I_{p} \\
C_{q}^{2} I_{p} \\
\vdots \\
C_{q}^{q} I_{p}
\end{array}\right)
$$

Assumption 1. The function $f(x, u)$ is globally Lipschitz with respect to $x(t)$, uniformly in $u(t)$.

$$
\left\|f\left(x_{1}, u\right)-f\left(x_{2}, u\right)\right\| \leq \gamma\left\|x_{1}-x_{2}\right\| .
$$

Under assumption (13), system (9) is an exponential observer for system (4), and the observation error converges asymptomatically to zero for quite high value of synthesis parameter $\theta>1$.

Furthermore, we present the following equations that establishes the dynamic error for a high gain fractional order class.
The estimation error of ${ }^{c} D_{t_{0}}^{\alpha} e=D_{t_{0}}^{\alpha} \hat{x}-D_{t 0}^{\alpha} x$ is substituted into the corresponding dynamic equations (4) and (9):

$$
{ }^{c} D_{t_{0}}^{\alpha} e=A(\widehat{x}-x)-\theta \Delta_{\theta}^{-1} S^{-1} C^{T}(C \widehat{x}-y)+f(\widehat{x}, u)-f(x, u) .
$$

So, we get

$$
{ }^{c} D_{t_{0}}^{\alpha} e=\left(A-\theta \Delta_{\theta}^{-1} S^{-1} C^{T} C\right) e+f(\widehat{x}, u)-f(x, u) .
$$

We introduce the following change of variable $\bar{e}(t)=\Delta_{\theta} e(t)$, and we obtain

$$
{ }^{c} D_{t_{0}}^{\alpha} \bar{e}=\left(A-\theta S^{-1} C^{T} C\right) \bar{e}+\Delta_{\theta}(f(\widehat{x}, u)-f(x, u)) .
$$

Remark 1. More specifically, the high-gain observers have design flexibility and can be easily adapted to the design of adaptive fuzzy observers proposed by Boulkroune et al. in [30]. In the same way, numerical approximations show a high degree of convergence, which indicates that the results are much closer to the actual.

\section{Stability of the Proposed High-Gain Observer}

We defined the stability of the system (9) which ensures the Mittag-Leffler stability, the Lyapunov function is checked as $V_{\theta}(\bar{e}, t)=\bar{e}^{T}(t) \operatorname{Se}(t)>0$ such that $V_{\theta}(0)=0$ with its derivatives along the trajectories of ${ }^{c} D_{t_{0}}^{\alpha} e$ :

$$
\begin{gathered}
\lambda\|e\|^{p} \leq V(e(t)), \\
{ }_{0}^{c} D_{t}^{\alpha} V(e(t)) \leq-\gamma V(e(t)),
\end{gathered}
$$

where $\lambda>0, p>0$, and $\gamma>0$; then, fractional-order system (9) is a Mittag-Leffler high-gain observer for system (4) and $\|\widehat{x}-x\| \leq k[E(-\gamma t)]^{1 / p}$ for all $t \geq 1$, where $k$ is a positive number based on $\hat{x}_{0}-x_{0}$.

Therefore, from expression (16) and Lemma 1, the fractional Caputo derivative of the Lyapunov function can be defined as follows:

$$
{ }^{c} D_{t_{0}}^{\alpha} V_{\theta}(\bar{e}, t) \leq-\theta \bar{e}^{T} S \bar{e}+(1-2 \theta) \bar{e}^{T} C^{T} C \bar{e}+2 \bar{e}^{T} S \Delta_{\theta}(f(\widehat{x}, u)-f(x, u)) .
$$

Proof. We have the following expression:

$$
{ }^{c} D_{t_{0}}^{\alpha} V_{\theta}(\bar{e}, t)=2 \bar{e}^{T}(t) S^{c} D_{t_{0}}^{\alpha} \bar{e}(t)
$$

$$
V_{\theta}(\bar{e}, t)=\bar{e}^{T}(t) \operatorname{Se}(t) .
$$

and then

So,

$$
\begin{aligned}
{ }^{c} D_{t_{0}}^{\alpha} V_{\theta}(e, t) & =\bar{e}^{T}\left[\left(A-\theta S^{-1} C^{T} C\right)^{T}+\Delta_{\theta}^{T} K(\cdot)^{T}\right] S \bar{e}+\bar{e}^{T} S\left[\left(A-\theta S^{-1} C^{T} C\right) \bar{e}+\Delta_{\theta} K(\cdot)\right], \\
K(\cdot) & =(f(\hat{x}, u)-f(x, u)) .
\end{aligned}
$$


In addition,

$$
{ }^{c} D_{t_{0}}^{\alpha} V_{\theta}(\bar{e}, t) \leq \bar{e}^{T}\left[\left(A-\theta S^{-1} C^{T} C\right)^{T} S+S\left(A-\theta S^{-1} C^{T} C\right)\right] \bar{e}+2 \bar{e}^{T} S \Delta_{\theta} K(\cdot),
$$

or

${ }^{c} D_{t_{0}}^{\alpha} V_{\theta}(\bar{e}, t) \leq \bar{e}^{T}\left(A^{T} S-2 \theta S^{-1} C^{T} C+S A\right) \bar{e}+2 \bar{e}^{T} S \Delta_{\theta} K(\cdot)$.

Finally, when using (11), we found

$$
\begin{aligned}
{ }^{c} D_{t_{0}}^{\alpha} V_{\theta}(\bar{e}, t) & \leq-\theta \bar{e}^{T} S \bar{e}+(1-2 \theta) \bar{e}^{T} C^{T} C \bar{e}+2 \bar{e}^{T} S \Delta_{\theta} K(\cdot), \\
{ }^{c} D_{t_{0}}^{\alpha} V_{\theta}(e) & \leq-\theta e^{T} S e+(1-2 \theta) e^{T} \Delta_{\theta} C^{T} C e+2\|e\|\|S\|\|K(\cdot)\| .
\end{aligned}
$$

End of proof [13].

Using Assumption 1, we deduce that

$$
\left\|\Delta_{\theta}(f(\widehat{x}, u)-f(x, u))\right\| \leq \gamma\|e\|
$$

for $\theta \geq 1$. And if condition (17) is replaced by

$$
\begin{aligned}
\lambda_{1}\|e\|^{2} & \leq V(e(t)) \leq \lambda_{2}\|e\|^{2}, \\
V(e(t)) & \leq \lambda_{2}\|e\|^{2} .
\end{aligned}
$$

Hence, from expression (25), equation (18) becomes

$$
\begin{gathered}
{ }^{c} D_{t_{0}}^{\alpha} V_{\theta}(e) \leq-\lambda_{\min }\left(\theta S+(2 \theta-1) \Delta_{\theta} C^{T} C\right)\|e\|^{2}+2 \gamma \lambda_{\max }\|e\|^{2} \\
\gamma \prec \frac{\lambda_{\min }\left(\theta S+(2 \theta-1) \Delta_{\theta} C^{T} C\right)}{2 \lambda_{\max }(S)} .
\end{gathered}
$$

Then, from (17), we get

$$
V(e(t)) \leq-d\|e\|^{2}, \quad \text { with } d=\lambda_{\min }\left(\theta S+(2 \theta-1) \Delta_{\theta} C^{T} C\right)-2 \gamma \lambda_{\max }(S)>0 .
$$

So, if $\gamma \prec\left(\left(\lambda_{\min }\left(\theta S+(2 \theta-1) \Delta_{\theta} C^{T} C\right)\right) / 2 \lambda_{\max }(S)\right)$ and Theorem 1 and Assumption 1 are satisfied, then the fractional-order high-gain observer converges exponentially, which is globally Mittag-Leffler stable.

Assumption 2. There exists a matrix $k$ such that $\operatorname{Re}(\lambda(A+B K)) \prec 0$, the pair $(\mathrm{A}, \mathrm{B})$ is stabilizable, and a Lyapunov function $V(x)=x^{T} P x$, where $P=P^{T}>0$ such that

$$
P(A+B K)+(A+B K)^{T}, \quad P=-Q, Q>0 .
$$

Proof. The derivate of $V(x)$ satisfies

$$
{ }^{c} D_{t_{0}}^{\alpha} V_{\theta}(x) \leq-x^{T} Q x+2 x^{T} P f(x, k x) .
$$

Using Assumption 1 and $f(0, u)=0$,

$$
{ }^{c} D_{t_{0}}^{\alpha} V_{\theta}(x) \leq\left[-\lambda_{\min }(Q)+2 \gamma \lambda_{\max }(P)\right]\|x\|^{2} .
$$

Then, the Mittag-Leffler feedback law $u(x)=k x$ stabilized system (4) when Assumptions 2 and 1 are verified, and the Lipschitz constant $\gamma$ verifies

$$
\gamma \prec \frac{\lambda_{\min }(Q)}{2 \lambda_{\max }(P)} \text {. }
$$

Theorem 2. If (11) and Assumptions 2 and 1 hold and if

$$
\gamma \prec \frac{1}{2} \inf \left(\frac{1}{\lambda_{\max }(P)}, \frac{\lambda_{\min }\left(\theta S+(2 \theta-1) \Delta_{\theta} C^{T} C\right)}{2 \lambda_{\max }(S)}\right), \quad \theta \geq 1
$$

then the system

$$
\left\{{ }^{c} D_{t_{0}}^{\alpha} \hat{x}(t),{ }^{c} D_{t_{0}}^{\alpha} e(t),\right.
$$

is globally Mittag-Leffler stable.

The feedback controller is synthesized under sufficient conditions of linear matrix inequalities and expressed in terms of Riccati differential equations. But in this paper, we use a parameter to prove the global asymptotic stability of the nonlinear system.

\section{Simulation Results}

In order to evaluate the performance of the proposed observer, two simulation experiments were carried out for this study.

We performed a comparative study between the proposed approach and the developed method [22, 31] with a variation of $\alpha$ value and using the predictor-corrector (PC) method.

5.1. First Example of Fractional-Order System. The fractional-order system used in [31] given by equation (35) is examined. 


$$
\begin{aligned}
{ }^{c} D_{t_{0}}^{\alpha} x_{1}(t) & =3 x_{2}+u+0.2 \sin \left(x_{2}\right) \sin (u), \\
{ }^{c} D_{t_{0}}^{\alpha} x_{2}(t) & =x_{1}+0.2 \sin \left(x_{1}\right), \quad t \geq t_{0}, \\
y & =C x=x_{1}, \\
t & \geq t_{0},
\end{aligned}
$$

with $u=-x_{1}-4 x_{2}, A=\left[\begin{array}{ll}0 & 3 \\ 1 & 0\end{array}\right], B=\left[\begin{array}{l}1 \\ 0\end{array}\right]$, and $f(x, u)$ $=0.2\left[\begin{array}{c}\sin \left(x_{2}\right) \sin (u) \\ \sin \left(x_{1}\right)\end{array}\right]$

According to the general expression of the fractional high-gain observer given by expression (9), we proposed the FHGO of system (35) as follows:

$$
\begin{aligned}
{ }^{c} D_{t_{0}}^{\alpha} \widehat{x}_{1}(t)= & 3 \widehat{x}_{2}+\widehat{u}+0.2 \sin \left(\widehat{x}_{2}\right) \sin (\widehat{u})-2 \theta \times\left(\widehat{x}_{1}-x_{1}\right), \\
{ }^{c} D_{t_{0}}^{\alpha} \widehat{x}_{2}(t)= & \widehat{x}_{1}+0.2 \sin \left(\widehat{x}_{1}\right), \\
& -\theta^{2} \times\left(3+0.2 \times \cos \left(\hat{x}_{2}\right) \sin (\widehat{u})\right) \times\left(\hat{x}_{1}-x_{1}\right), \\
y= & C \hat{x}=\widehat{x}_{1} .
\end{aligned}
$$

Moreover, using $\theta=7$, the Lipchitz constant verifies $\gamma=0.2 \prec\left(\left(\lambda_{\min }\left(\theta S+(2 \theta-1) \Delta_{\theta} C^{T} C\right)\right) / 2 \lambda_{\max }(S)\right) \approx 0.99$.

The pair $(\mathrm{A}, \mathrm{C})$ is observable, and by solving expression (11), using LMI MATLAB, we obtain

$$
S=\left[\begin{array}{cc}
0.1597 & -0.0628 \\
-0.0628 & 0.0707
\end{array}\right]=S^{T}>0 .
$$

Applying conditions given by expressions (11), (13), and (27), the system given by expression (35) can be globally Mittag-Leffler stabilizable.

On the other hand, by solving expression in Assumption 2, we find

$$
P=\left[\begin{array}{ll}
0.4 & 0.1 \\
0.1 & 0.3
\end{array}\right]=P^{T}>0
$$

Furthermore, the Lipschitz constant verifies $\gamma=0.2 \prec\left(\lambda_{\text {min }}(Q) / 2 \lambda_{\text {max }}(P)\right) \approx 0.92$. So, the controlled system is globally Mittag-Leffler stable. This validates the theoretical results. This concludes the proof of Theorem 2 .

Now, using the initial conditions as $x_{0}=\left[\begin{array}{ll}-1.5 & 1\end{array}\right]^{T}$, $\widehat{x}_{0}=\left[\begin{array}{ll}-1 & 2\end{array}\right]^{T}$, and $\alpha=0.5$, Figures 1 and 2 illustrate the obtained results.

From these figures, it is noticed that the proposed FHGO design approach gives a good concordance with the state system. Compared with the results obtained in [31], we can understand that the proposed design of fractional high-gain observer achieves the same performance as the observer used in [31], with better convergence error. One can notice that system (35) is Mittag-Leffler stable, and the estimation error asymptotically tends towards zero, which implies asymptotic stability as given by Figure 3.

Furthermore, our objective, in this paper, is to study the change in the value of order $\alpha$ and their influence on the FHGO performances; Figures 4 and 5 are investigated for different values of $\alpha(\alpha=0.6, \alpha=0.9)$.

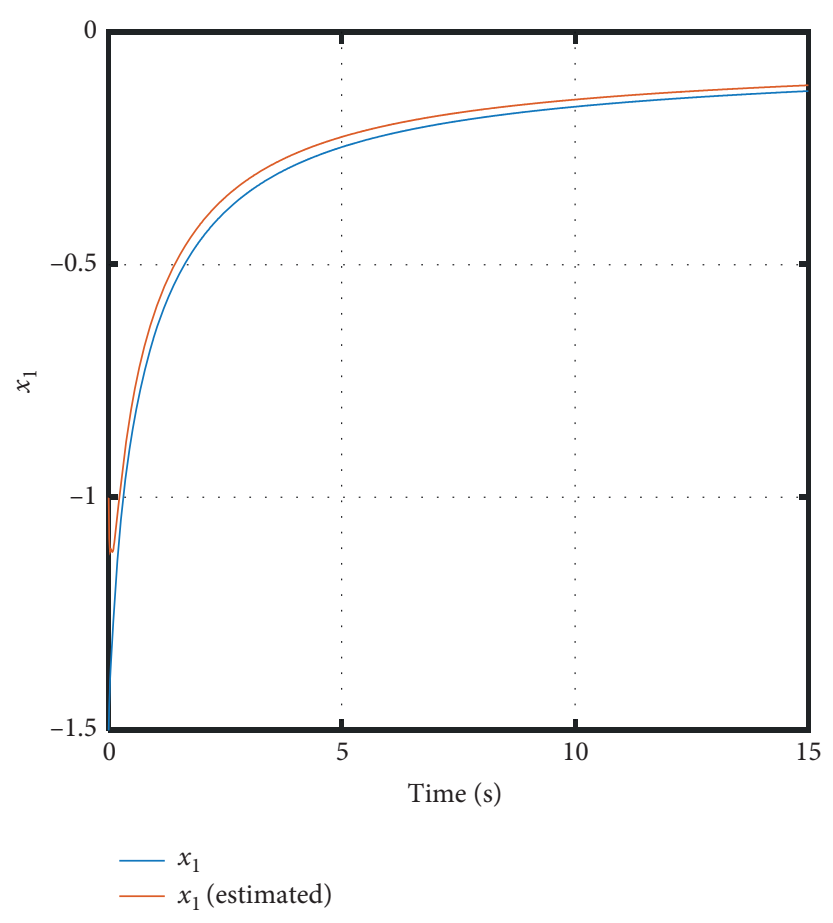

Figure 1: $x_{1}$ and $\hat{x}_{1}$ for $\alpha=0.5$.

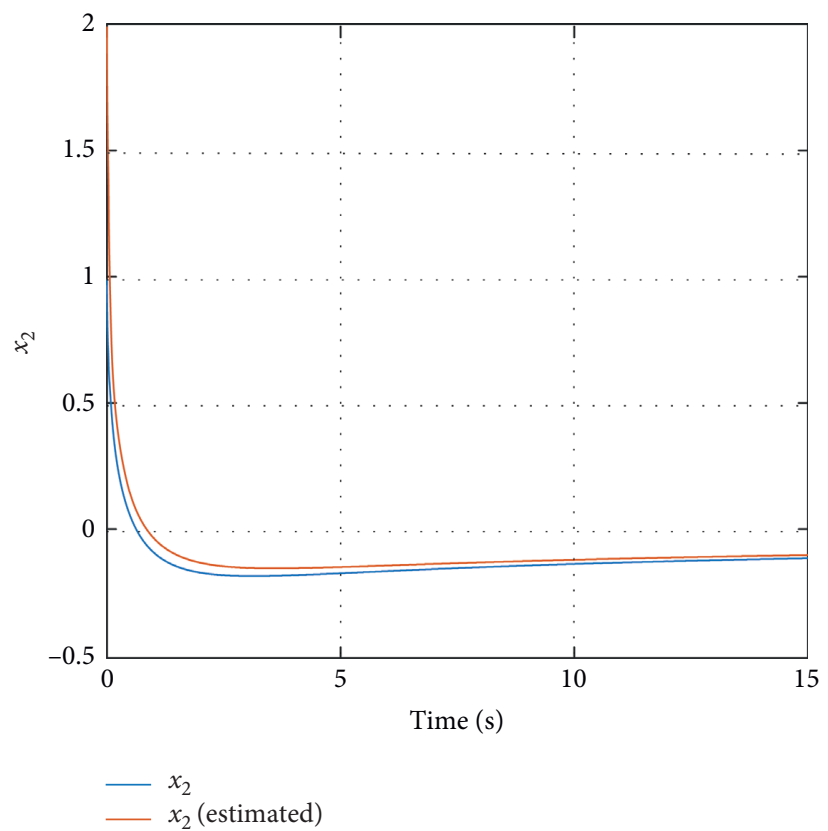

Figure 2: $x_{2}$ and $\hat{x}_{2}$ for $\alpha=0.5$.

From simulation results presented in Figures 3 and 4, it is shown that for each value of $\alpha$, the dynamics of the system change. The proposed FHGO presents good results in each case of $\alpha$. It is noticed that when $\alpha=0.9$, the system is faster than the case of $\alpha=0.6$.

Hence, this modification in the value of $\alpha$ makes the estimate more consistent with the current state. 


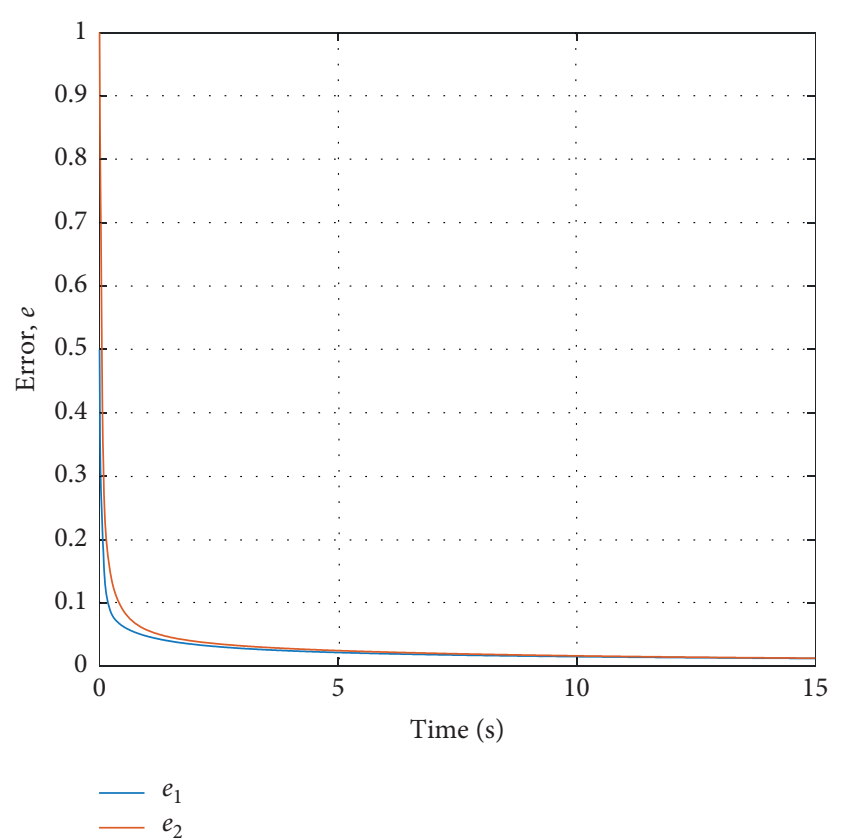

Figure 3: Convergence error.

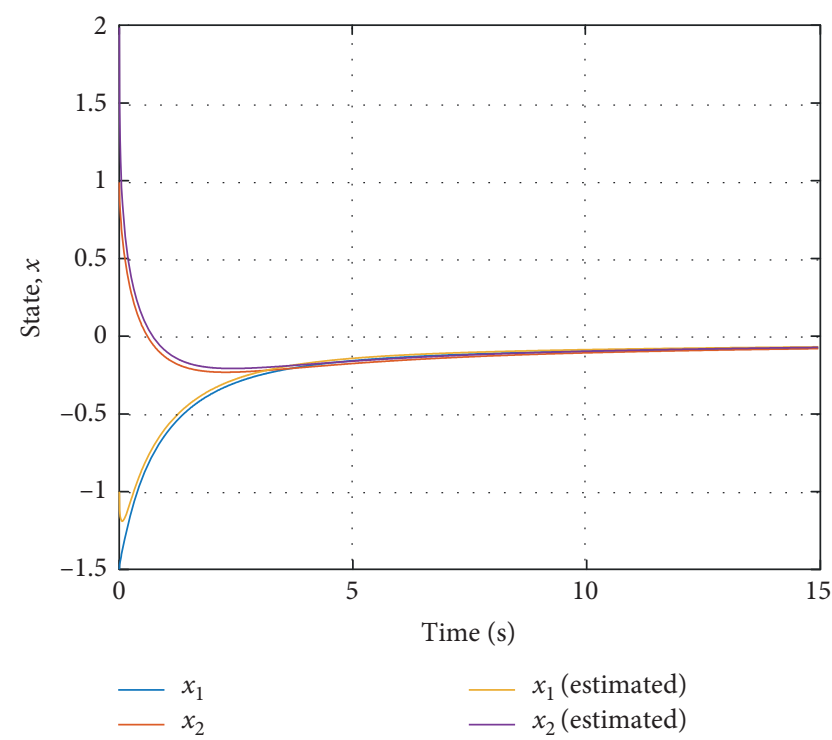

Figure $4: x_{1}, \hat{x}_{1}, x_{2}$, and $\hat{x}_{2}$ for $\alpha=0.6$.

With Figures 6 and 7, we can discuss the stability of system (35) when changing the $\alpha$ value.

So, in Figure 7, it is observed that the estimation error converges more rapidly to zero when $\alpha=0.9$ than when we choose $\alpha=0.6$ in Figure 6 .

According to the stability conditions in the theory part, we have seen that they are satisfied in numerical results in Figures 6 and 7. Thus, the following condition is satisfied: $\|\widehat{x}-x\| \leq 0.2\left\|e_{0}\right\|\left[E_{\alpha}\left(\lambda\left(t-t_{0}\right)^{\alpha}\right)\right]^{(1 / 2)}$.

We can confirm that the controlled fractional-order system is asymptotically stable and that good control performance has been obtained.

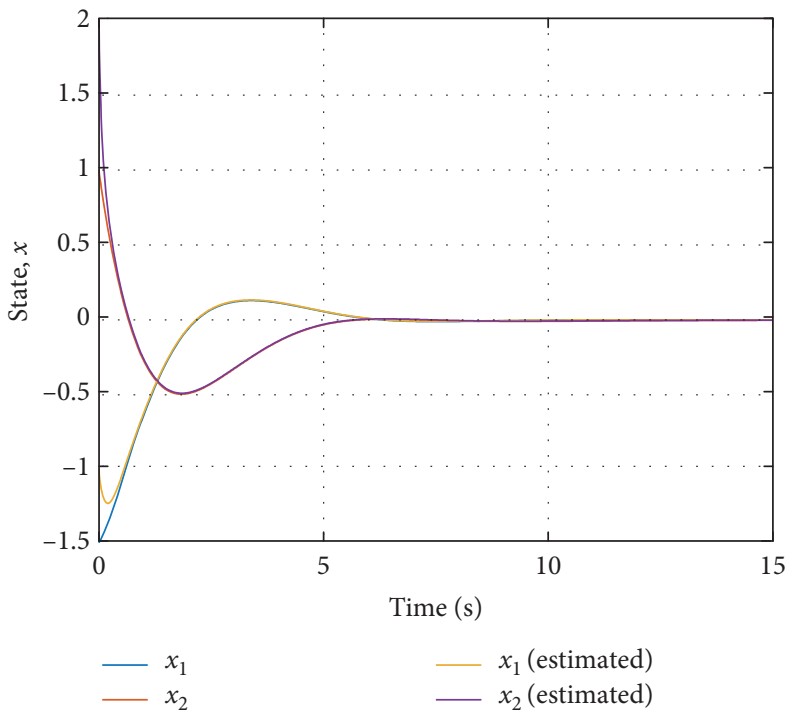

Figure 5: $x_{1}, \hat{x}_{1}, x_{2}$, and $\hat{x}_{2}$ for $\alpha=0.9$.

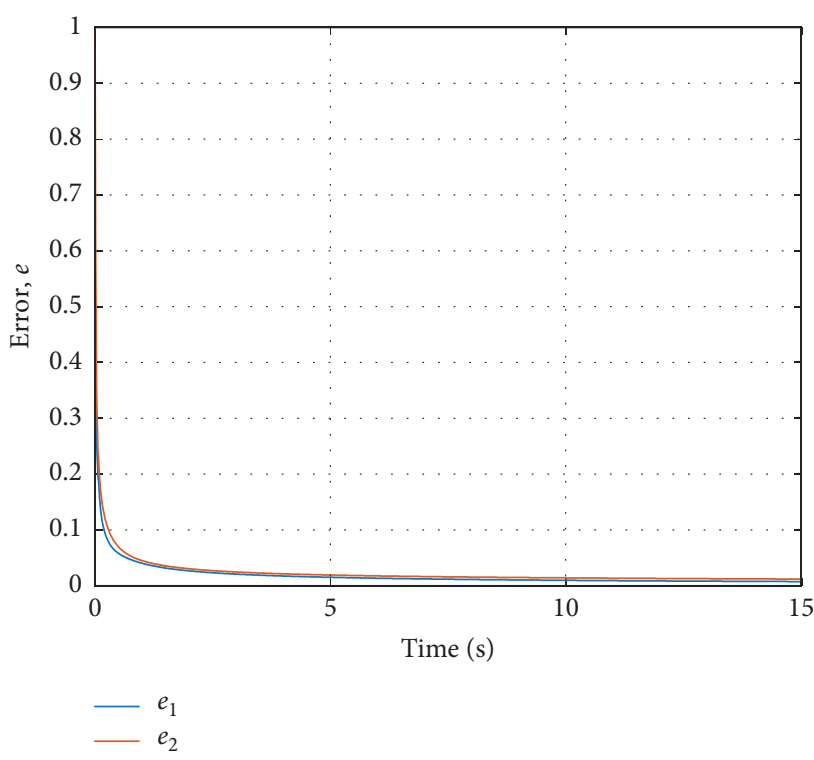

Figure 6: Estimation error when $\alpha=0.6$.

5.2. Second Example of Fractional-Order System. To examine the efficiency of the proposed FHGO approach, let us consider the fractional-order Arneodo system given by the following expression [22]:

$$
\begin{aligned}
{ }^{c} D_{t_{0}}^{\alpha} x_{1}(t) & =x_{2}, \\
{ }^{c} D_{t_{0}}^{\alpha} x_{2}(t) & =x_{3}, \\
{ }^{c} D_{t_{0}}^{\alpha} x_{3}(t) & =-5.5 x_{1}-3.5 x_{2}-0.25 x_{3}-x_{1}^{3}, \\
y & =C x=x_{1} .
\end{aligned}
$$

The fractional high-gain observer of system (39) obtained from the theoretical calculations is illustrated by the following equation: 

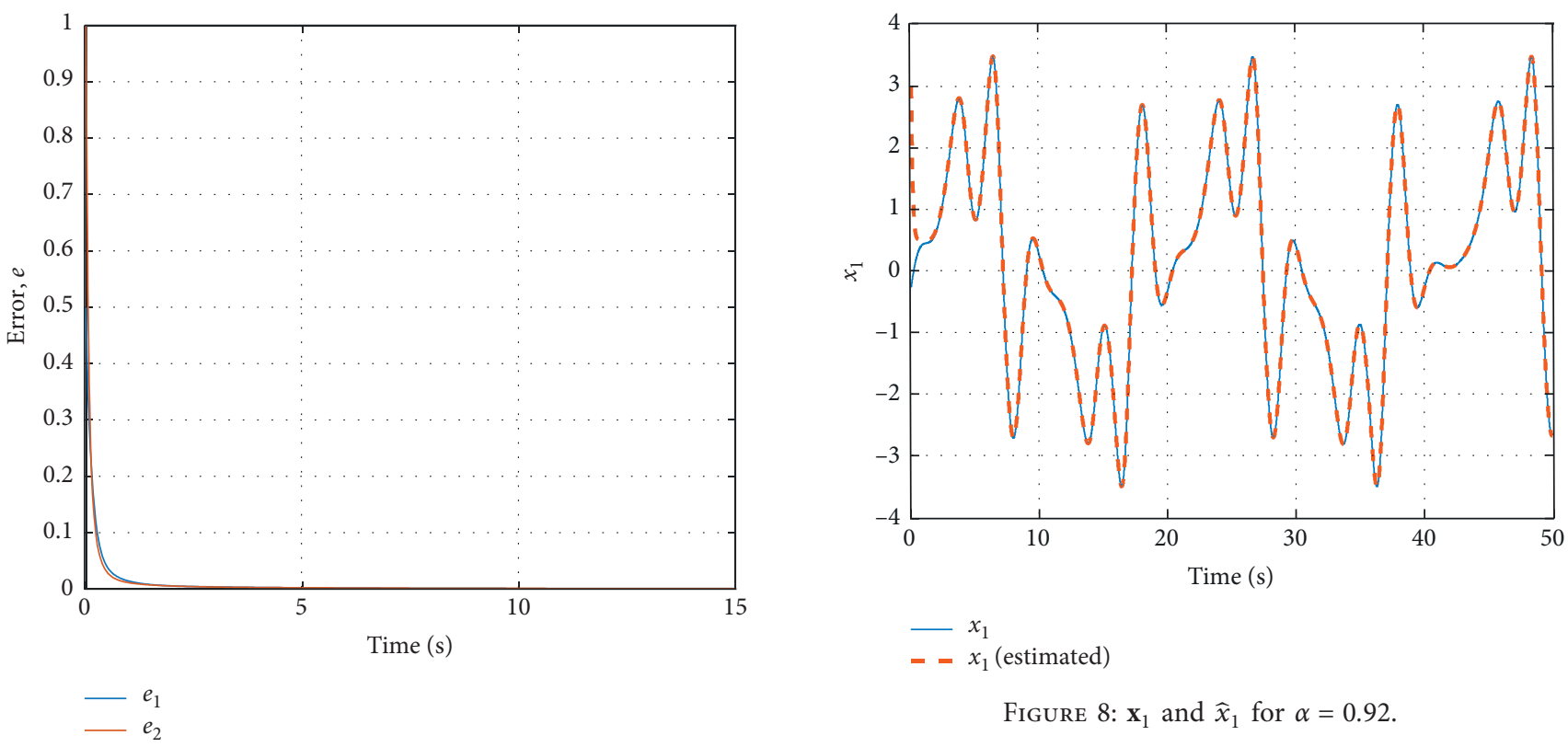

FIGURE 7: Estimation error when $\alpha=0.9$.

$$
\begin{aligned}
{ }^{c} D_{t_{0}}^{\alpha} \hat{x}_{1}(t) & =\widehat{x}_{2}-3 \theta\left(\hat{x}_{1}-x_{1}\right), \\
{ }^{c} D_{t_{0}}^{\alpha} \widehat{x}_{2}(t) & =x_{3}-3 \theta^{2}\left(\widehat{x}_{1}-x_{1}\right), \\
{ }^{c} D_{t_{0}}^{\alpha} \hat{x}_{3}(t) & =5.5 \hat{x}_{1}-3.5 \hat{x}_{2}-0.25 \widehat{x}_{3}-\hat{x}_{1}^{3}-\theta^{3}\left(\hat{x}_{1}-x_{1}\right), \\
\hat{y} & =C \hat{x}=\hat{x}_{1} .
\end{aligned}
$$

By using the initial conditions as $x_{0}=\left[\begin{array}{lll}-0.25 & 1.2 & 0.2\end{array}\right]^{T}, \quad \alpha=0.92, \quad \theta=20, \quad$ and $\widehat{x}_{0}=\left[\begin{array}{lll}3 & 1.8 & 0.25\end{array}\right]^{T}$ and applying the PC method, the following simulations are presented (Figures 8-10).

The results show that the estimated states $\widehat{x}_{1}, \hat{x}_{2}$, and $\hat{x}_{3}$ converge to the current states $x_{1}, x_{2}$, and $x_{3}$.

It is found that the high gain fractional observer (40) achieves fast and stable convergence estimation. It can be noted that the proposed approach has a high performance.

From Figure 11, we can notice that the estimation convergence error tends towards the origin state. Thus, system (40) is stable.

Compared with the results obtained in [22], we can remark that the fractional high-gain observer achieves better performance than the observer used in [22], as designed to provide good state estimates and especially $x_{2}$ and $x_{2}$.

Since the Arneodo system is defined as a chaotic system, the results have shown the efficiency of the proposed method on the fractional-order chaotic systems.

Moreover, Figures 12 and 13 illustrate the influence of $\alpha$ on the Arneodo system.

In Figures 12 and 13, we can observe the dynamics of the Arneodo system, which has lost synchronization in time when we decrease alpha to zero $(0<\alpha<1)$. We see that even though the amplitudes of the dynamics system are changed, the observer has good tracking performance and estimation.
Figure 8: $\mathbf{x}_{1}$ and $\hat{x}_{1}$ for $\alpha=0.92$.

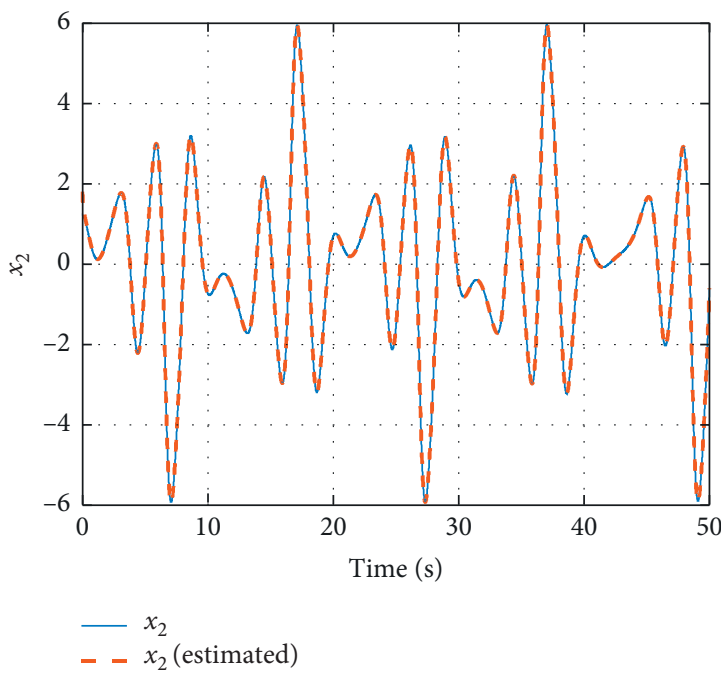

Figure 9: $\mathbf{x}_{2}$ and $\widehat{x}_{2}$ for $\alpha=0.92$.

These comparisons are made to show how the states and their estimates are changed when the fractional order is modified (0.92, 0.8, and 0.7$)$, and this undoubtedly improves the performance.

From Figures 14 and 15, we can conclude that $\| \hat{x}-$ $x \| \longrightarrow 0$ when $t \longrightarrow \infty$. So, the condition $\|\hat{x}-x\| \leq \sqrt{\left(\lambda_{\max }(S) / \lambda_{\min }(S)\right)}\left\|\widehat{x}_{0}-x_{0}\right\|\left[E_{\alpha}\left(\lambda\left(t-t_{0}\right)^{\alpha}\right)\right]^{(1 / 2)}$ is satisfied, and the Mittag-Leffler stability is proved.

In more detail, Figures 14 and 15 show that the error converges to zero such that $e_{2}$ converges faster than $e_{3}$ and $e_{1}$. Hence, the results of illustrative simulations of the synchronization of the fractional-order chaotic Arneodo system in the presence of different orders show good performance of this proposed observer.

We can show the efficiency of our proposed method during a perturbation, if we have an unknown external 


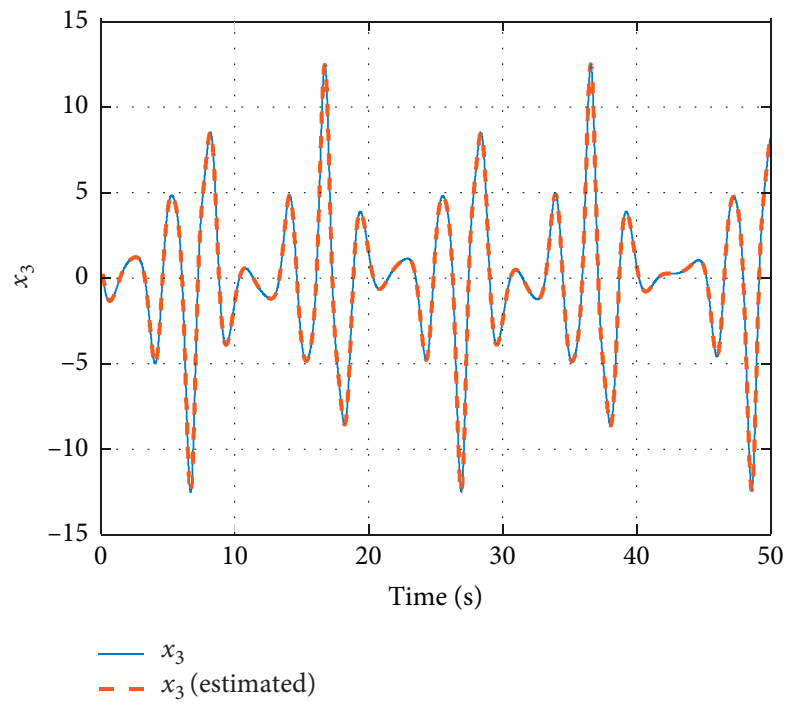

Figure 10: $\mathbf{x}_{3}$ and $\widehat{x}_{3}$ for $\alpha=0.92$.

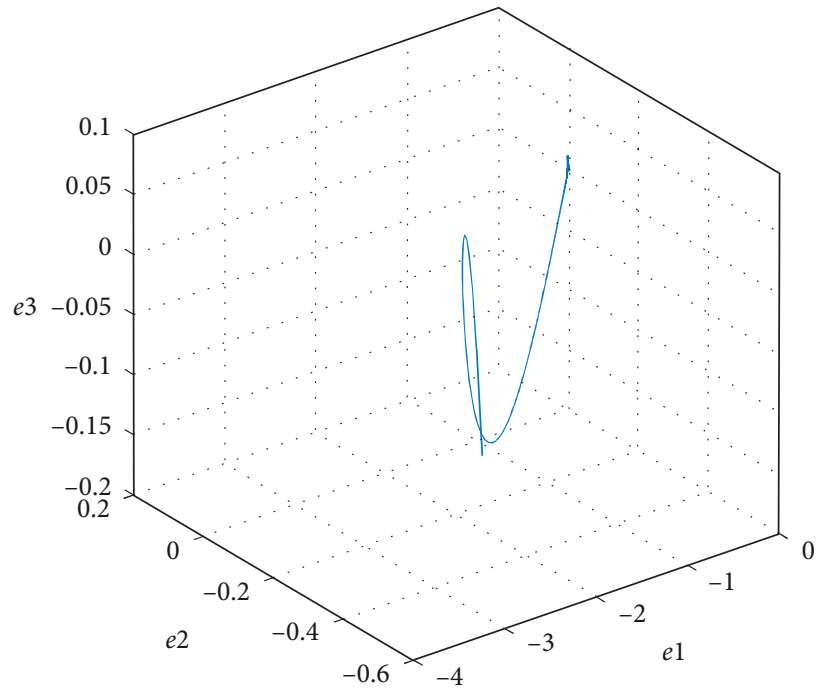

FIGURE 11: Estimation convergence error.

disturbance presented in the state $x_{3}$, taken as $g(t)=0.5 \cos (5 t) x_{3}-0.5 \sin (t)$.

The equation of system (39) becomes

$$
\begin{aligned}
{ }^{c} D_{t_{0}}^{\alpha} x_{1}(t) & =x_{2}, \\
{ }^{c} D_{t_{0}}^{\alpha} x_{2}(t) & =x_{3}, \\
{ }^{c} D_{t_{0} x_{3}}^{\alpha}(t) & =-5.5 x_{1}-3.5 x_{2}-0.25 x_{3}-x_{1}^{3}+g(t), \\
y & =C x=x_{1} .
\end{aligned}
$$

The simulation results are shown in Figure 16.
In Figure 16, we see that even though the disturbance is presented, the proposed method has a good tracking performance.

The high-gain observer is robust in modelling uncertainties and external disturbances and guarantees a fast exponential decay of the estimation error.

We consider the measurement noise in the output of the system; we add white Gaussian noise to signal.

In Figure 17, it is observed that the algorithm (40) provides fast convergence even in the presence of noise that affects the available output signal. 

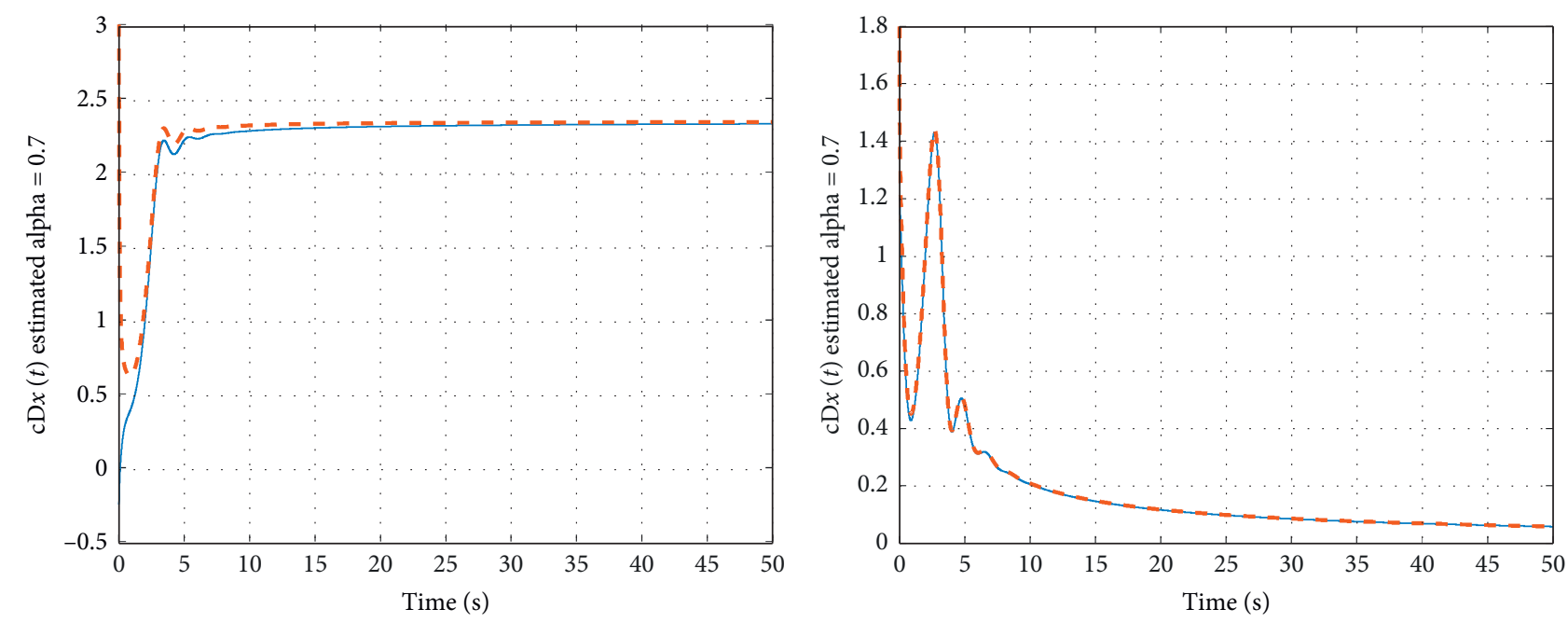

$-x_{1}$
$--x_{1}$ (estimated)

$-x_{2}$
$--x_{2}($ estimated $)$

(a)

(b)

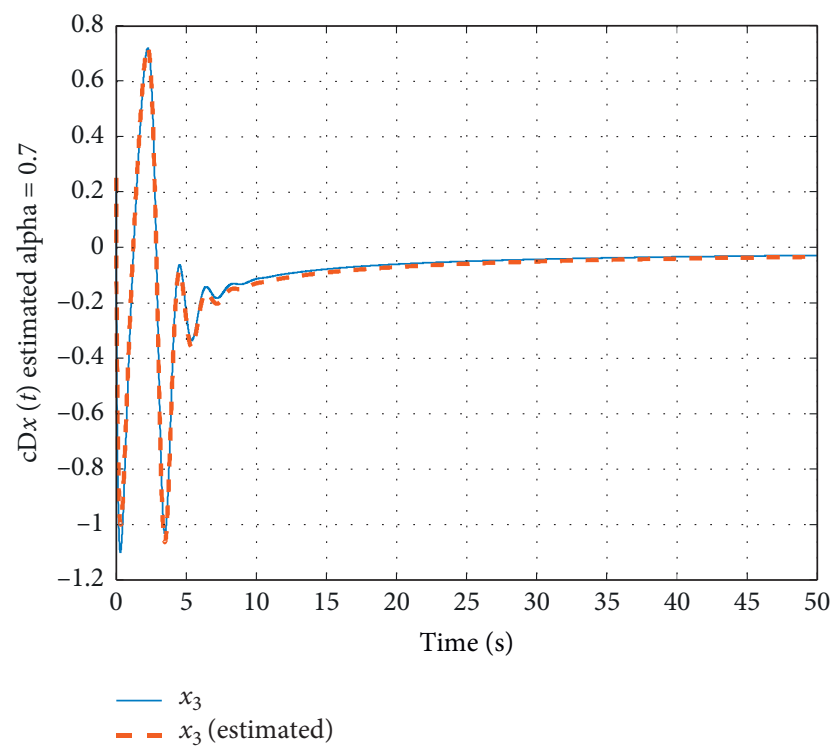

(c)

FIgURE 12: The estimated states with $\alpha=0.7$. 

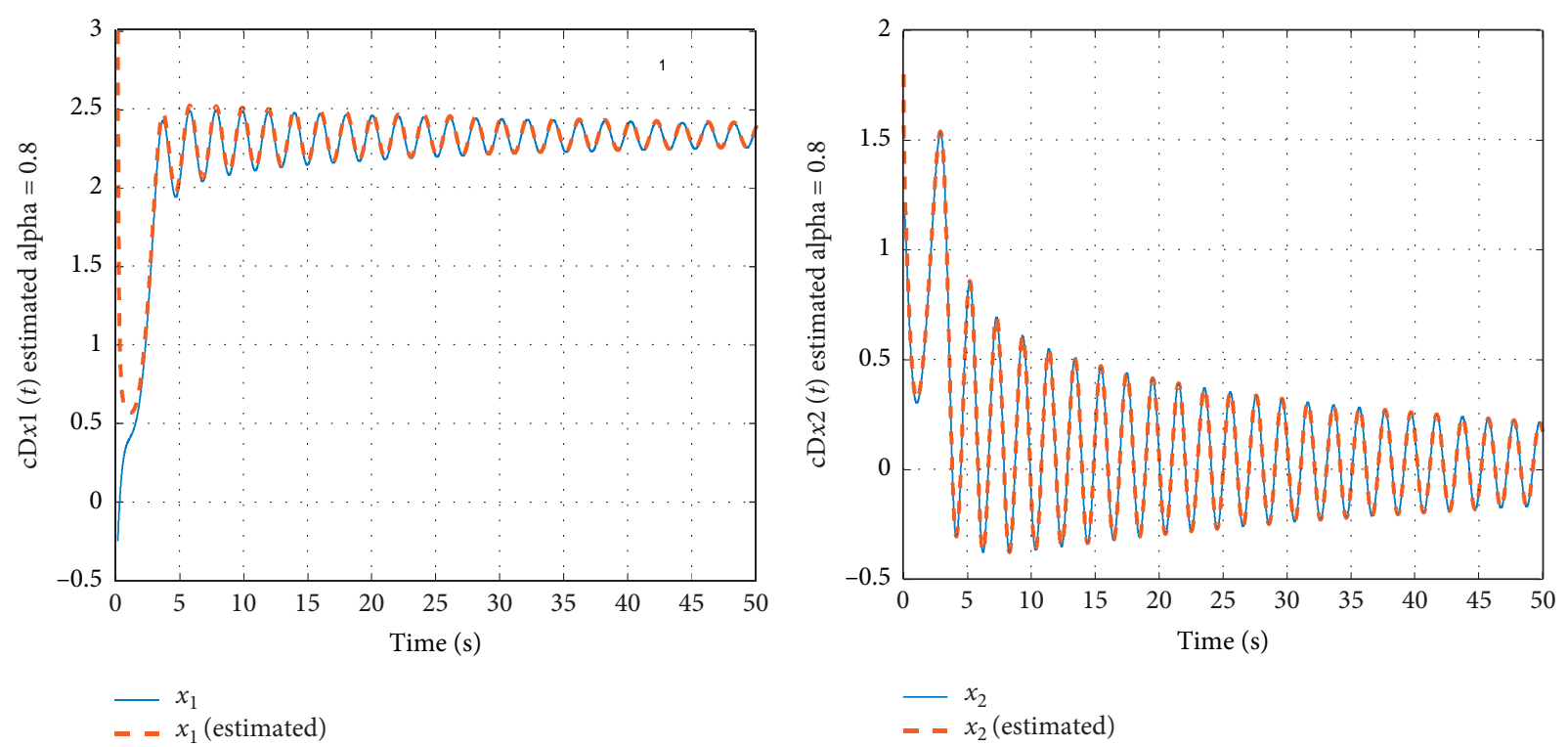

(a)

(b)

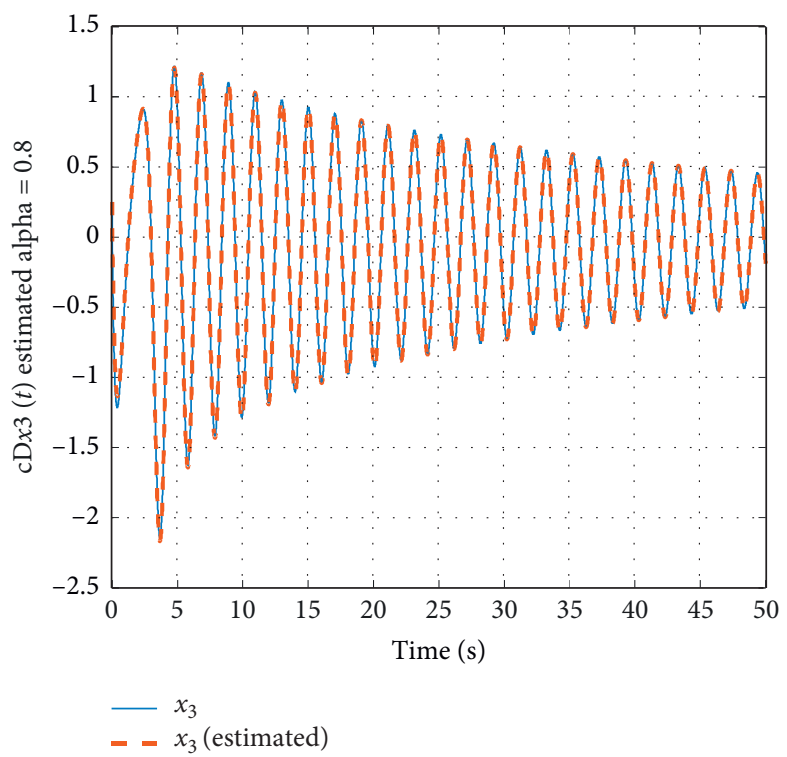

(c)

Figure 13: The estimated states with $\alpha=0.8$. 


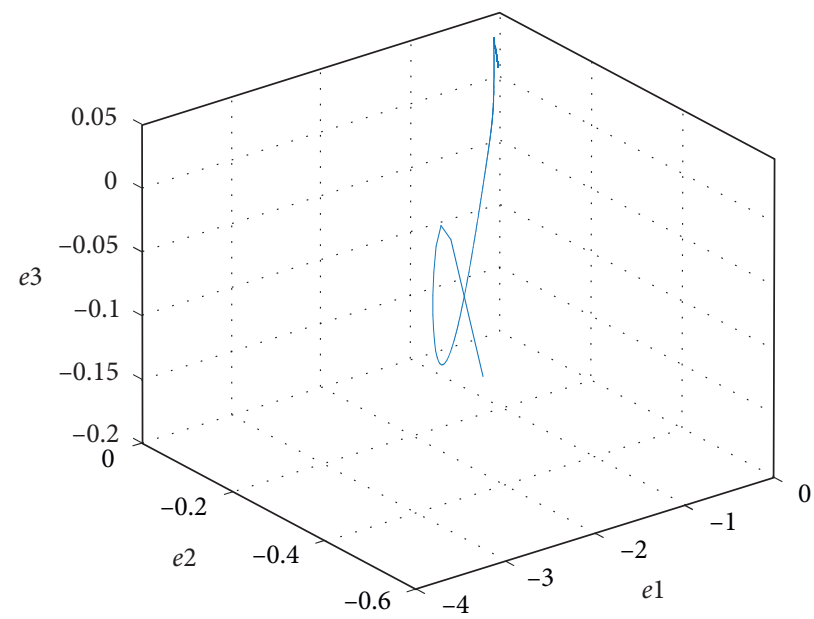

Figure 14: Convergence error for $\alpha=0.7$.

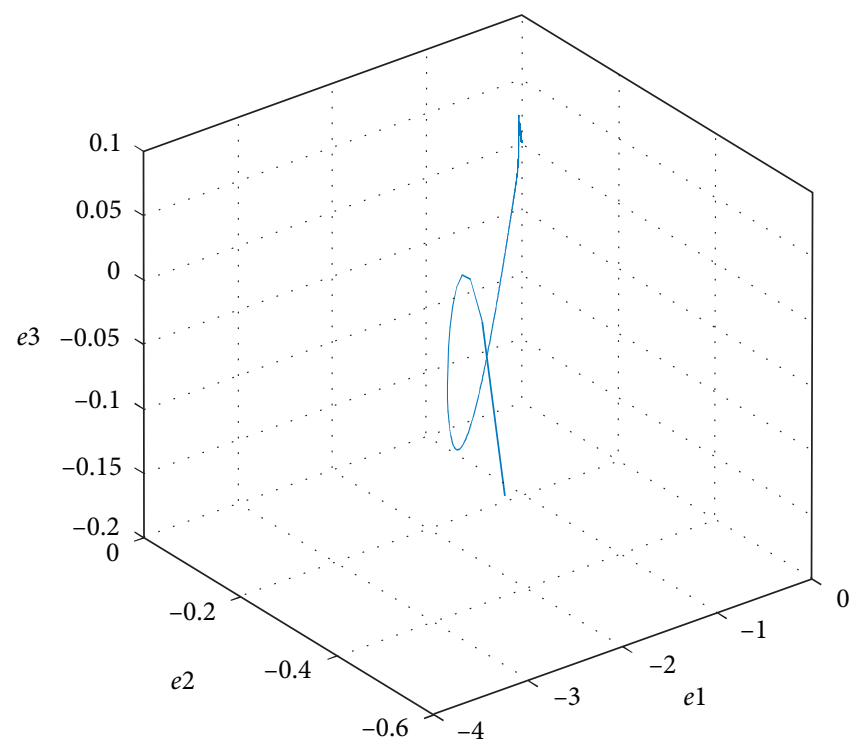

Figure 15: Convergence error for $\alpha=0.8$.
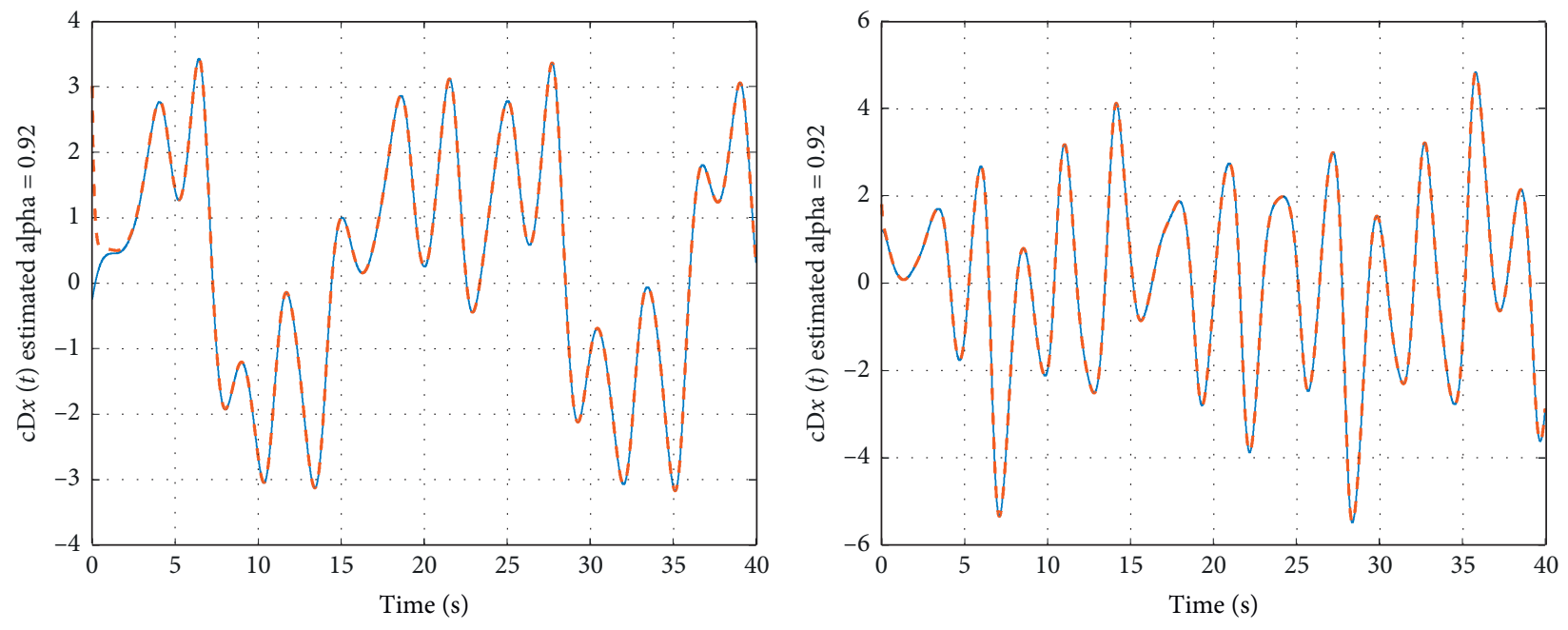

$-x_{1}$
$--x_{1}($ estimated $)$

$-x_{2}$
$--x_{2}$ (estimated)

(a) 


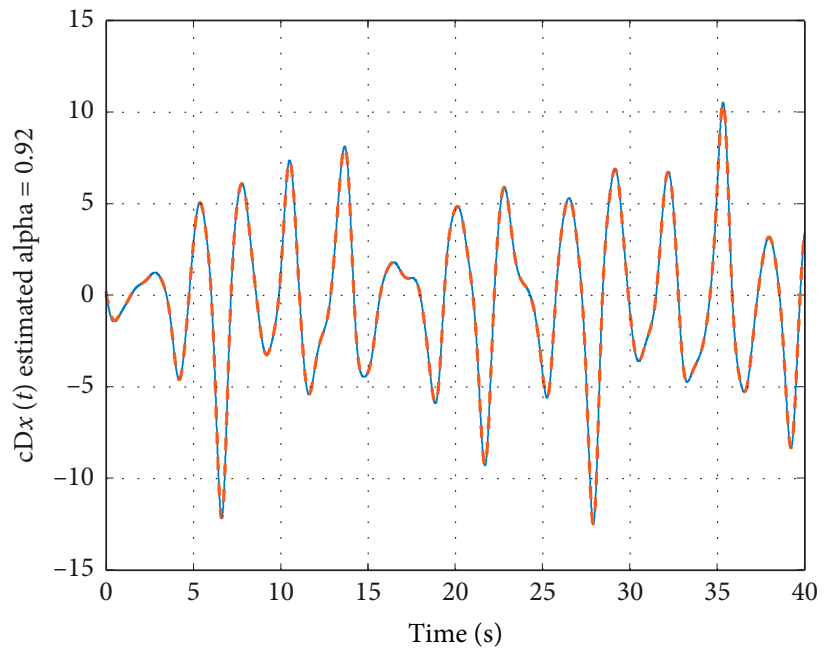

$-x_{3}$
$-\quad x_{3}($ estimated $)$

(c)

FIGURE 16: Estimation of fractional-order Arneodo system for $\alpha=0.92$ during the disturbance.
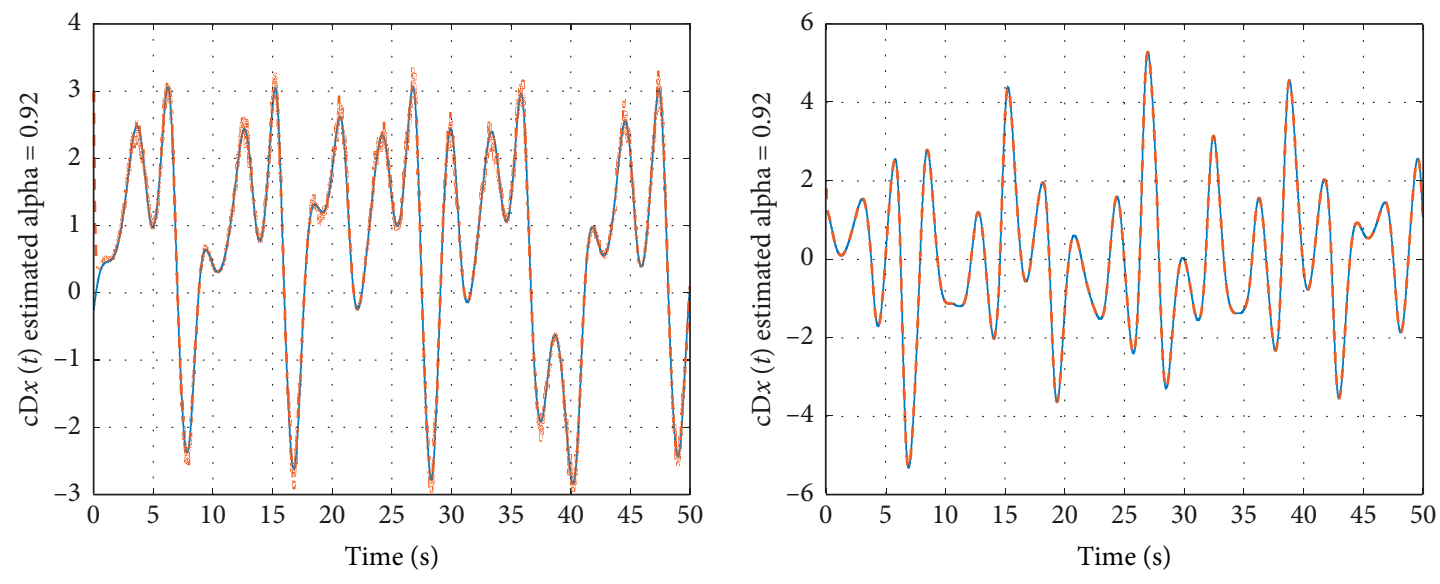

$\begin{array}{ll}- & x_{1} \\ - & x_{1}(\text { estimated })\end{array}$

$-x_{2}$
$-\quad x_{2}($ estimated $)$

(a)

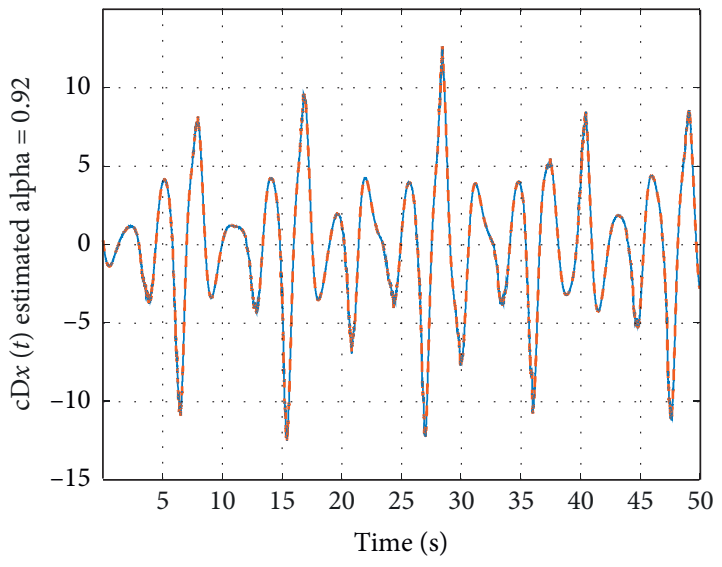

$-x_{3}$

$-x_{3}$ (estimated)

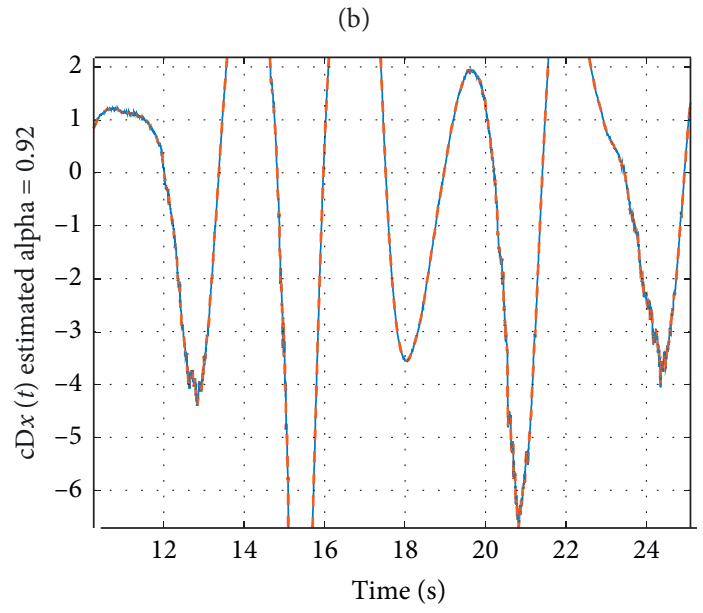

$x_{3}$
$--x_{3}($ estimated $)$

(c)

(d)

Figure 17: Estimation of Arneodo system for $\alpha=0.92$ with measurement noise in the output. 


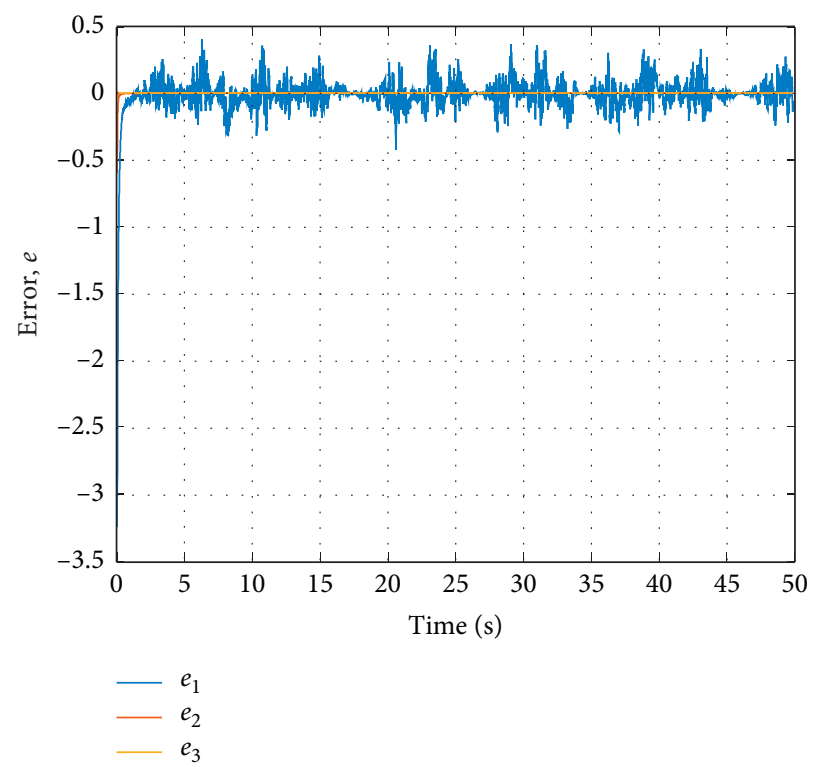

Figure 18: Estimation error of Arneodo system for $\alpha=0.92$ with measurement noise in the output.

Therefore, despite the modification of the fractional order, the observer provides a good convergence. The fractional high-gain observer has shown a good ability to reconstruct the system states, ensuring a low squared error compared to the classical observer.

It can be seen from Figure 18 that the approach considered provides a set of fractional laws that guarantee the asymptotic stability of fractional-order chaotic systems in the sense of the Lyapunov stability theorem.

\section{Conclusion}

In this paper, a fractional-order high-gain observer is designed for a class of nonlinear systems, which is characterized by their large performance. This approach allows fast convergence as the error converges to the origin. On the other hand, the system stability is examined, and the conditions that provide the Mittag-Leffler stability are shown.

This proposed approach is applied to two nonlinear fractional-order systems. According to the simulation results, this observer has good efficiency and the change of fractional-order value presents an interesting discussion. Indeed, every time we change the value of alpha, the dynamics and estimation of the system change.

Finally, we have verified the effectiveness of the proposed observer and its application to two examples, and they give good results. The results of illustrative simulations of the synchronization of the fractional-order chaotic Arneodo system in the presence of disturbance show the good performance and efficiency of this control solution.

\section{Data Availability}

The data used to support the findings of this study are included within the article.

\section{Conflicts of Interest}

The authors declare that they have no conflicts of interest.

\section{References}

[1] P. Ostalczyk, D. Sankowski, and J. Nowakowski, Non-integer Order Calculus and Its Applications, Springer, Berlin, Germany, 2018.

[2] C. Li, D. Qian, and Y. Chen, "On Riemann-Liouville and Caputo derivatives," Discrete Dynamics in Nature and Society, vol. 2011, Article ID 562494, 15 pages, 2011.

[3] R. Hilfer, Y. Luchko, and Z. Tomovski, "Operational method for the solution of fractional differential equations with generalized Riemann-Liouville fractional derivatives," Fractional Calculus and Applied Analysis, vol. 12, no. 3, pp. 299-318, 2009.

[4] M. D. Ortigueira, L. Rodríguez-Germá, and J. J. Trujillo, "Complex Grünwald-Letnikov, Liouville, Riemann-Liouville, and Caputo derivatives for analytic functions," Communications in Nonlinear Science and Numerical Simulation, vol. 16, no. 11, pp. 4174-4182, 2011.

[5] Y. B. Lahoucine Ouhsaine, M. El Ganaoui, M. Darouach, M. Zasadzinski, A. Mimet, and N. Eddine Radhy, "A general fractional-order heat transfer model for photovoltaic/thermal hybrid systems and its observer design," Energy Procedia, vol. 139, pp. 49-54, 2017.

[6] A. A. Kilbas, H. M. Srivastava, and J. J. Trujillo, Theory and Application of Fractional Differential Equations, Elsevier, Amsterdam, Netherlands, 2005.

[7] K. A. Abro, A. Siyal, and A. Atangana, "Thermal stratification of rotational second-grade fluid through fractional differential operators," Journal of Thermal Analysis and Calorimetry, vol. 143, no. 5, pp. 3667-3676, 2020.

[8] M. F. Tolba, A. M. AbdelAty, N. S. Soliman et al., "FPGA implementation of two fractional order chaotic systems," AEU-International Journal of Electronics and Communications, vol. 78, pp. 162-172, 2017. 
[9] A. M. Lopes, J. A. Tenreiro Machado, and A. M. Galhano, "Towards fractional sensors," Journal of Vibration and Control, vol. 25, no. 1, pp. 52-60, 2018.

[10] J. E. Solís-Pérez, J. F. Gómez-Aguilar, L. Torres, R. EscobarJiménez, and J. Reyes-Reyes, "Fitting of experimental data using a fractional Kalman-like observer," ISA Transactions, vol. 88, pp. 153-169, 2018.

[11] D. G. Luenberger, "Observing the state of a linear system," IEEE Transactions on Military Electronics, vol. 8, no. 2, pp. 74-80, 1964.

[12] A. Valibeygi, M. I. Hadi Balaghi, and K. Vijayaraghavan, "A comparative study of extended Kalman filter and an optimal nonlinear observer for state estimation," in Proceedings of the American Control Conference (ACC), Seattle, WA, USA, May 2017.

[13] C. Tréangle, M. Farza, and M. M’Saad, "Filtered high gain observer for a class of uncertain nonlinear systems with sampled outputs," Automatica, vol. 101, pp. 197-206, 2019.

[14] Y. Sun, J. Yu, Z. Li, and Y. Liu, "Coupled disturbance reconstruction by sliding mode observer approach for nonlinear system," International Journal of Control, Automation and Systems, vol. 15, no. 5, pp. 2292-2300, 2017.

[15] I. Elleuch, A. Khedher, and K. B. Othman, "State and faults estimation based on proportional integral sliding mode observer for uncertain Takagi-Sugeno fuzzy systems and its application to a turbo-reactor," International Journal of Fuzzy Systems, vol. 19, no. 6, pp. 1768-1781, 2017.

[16] J. G. Lu, "Nonlinear observer design to synchronize fractional-order chaotic systems via a scalar transmitted signal," Physica A, vol. 359, 2006.

[17] A. S. Balamash, M. Bettayeb, S. Djennoune, U. M. Al-Saggaf, and M. Moinuddin, "Fixed-time terminal synergetic observer for synchronization of fractional-order chaotic systems," Chaos: An Interdisciplinary Journal of Nonlinear Science, vol. 30, no. 7, 2020.

[18] S. L. Shaohua Luo, F. Tajaddodianfar, and J. Hu, "Observerbased adaptive stabilization of the fractional-order chaotic MEMS resonator," Nonlinear Dynamics, vol. 92, 2018.

[19] E. A. Boroujeni and H. R. Momeni, "Non-fragile nonlinear fractional order observer design for aclass of nonlinear fractional order systems," Signal Processing, vol. 92, no. 10, pp. 2365-2370, 2012.

[20] M. Bettayeb, U. M. Al-Saggaf, and S. Djennoune, "High gain observer design for fractional-order non-linear systems with delayed measurements: application to synchronisation of fractional-order chaotic systems," IET Control Theory \& Applications, vol. 11, no. 17, pp. 3171-3178, 2017.

[21] Z. Yu, Y. Zhang, Y. Qu, and Z. Xing, "Adaptive fractionalorder fault-tolerant tracking control for UAV based on highgain observer," in Proceedings of the International Design Engineering Technical Conferences and Computers and Information in Engineering Conference, August 2017.

[22] O. Martínez-Fuentes and R. Martínez-Guerra, "A high-gain observer with Mittag-Leffler rate of convergence for a class of nonlinear fractional-order systems," Communications in Nonlinear Science and Numerical Simulation, vol. 79, Article ID 104909, 2019.

[23] N. Djeghali, S. Djennoune, M. Bettayeb, M. Ghanes, and J.-P. Barbot, "Observation and sliding mode observer for nonlinear fractional-order system with unknown input," ISA Transactions, vol. 63, pp. 1-10, 2016.

[24] H. Delavari, H. Heydarinejad, and D. Baleanu, "Adaptive fractional-order blood glucose regulator based on high-order sliding mode observer," IET Systems Biology, vol. 13, no. 2, pp. 43-54, 2018.

[25] V. Ryali and K. M. Moudgalya, "Practical stability analysis of uncertain nonlinear systems," in Proceedings of the National Conference on Control and Dynamic Systems, IIT Bombay, Mumbai, India, January 2005.

[26] N. Aguila-Camacho, M. A. Duarte-Mermoud, and J. A. Gallegos, "Lyapunov functions for fractional order systems," Communications in Nonlinear Science and $\mathrm{Nu}$ merical Simulation, vol. 19, no. 9, pp. 2951-2957, 2014.

[27] Y.-H. Lan, H.-B. Gu, C.-X. Chen, Y. Zhou, and Y.-P. Luo, “An indirect Lyapunov approach to the observer-based robust control for fractional-order complex dynamic networks," Neurocomputing, vol. 136, pp. 235-242, 2014.

[28] Y. Li, Y. Chen, and I. Podlubny, "Mittag-Leffler stability of fractional order nonlinear dynamic systems," Automatica, vol. 45, no. 8, pp. 1965-1969, 2009.

[29] S. Dadras, S. Dadras, H. Malek, and Y. Q. Chen, "A note on the Lyapunov stability of fractional-order nonlinear systems," in Proceedings of the International Design Engineering Technical Conferences and Computers and Information in Engineering Conference, August 2017.

[30] A. Boulkroune, M. Tadjine, M. M'Saad, and M. Farza, “Design of a unified adaptive fuzzy observer for uncertain nonlinear systems," Information Sciences, vol. 265, pp. 139-153, 2014.

[31] A. Jmal, O. Naifar, A. Ben Makhlouf, N. Derbel, and M. A. Hammami, "On observer design for nonlinear caputo fractional-order systems," Asian Journal of Control, vol. 20, no. 4, pp. 1533-1540, 2018. 